\section{COOPERACIÓN PARA LA INVESTIGACIÓN, FORMACIÓN Y GESTIÓN TERRITORIAL: Universidad-Industria-Corporación de Desarrollo Regional ${ }^{1}$}

COOPERATION FOR TERRITORIAL RESEARCH, TRAINING

AND MANAGEMENT:

University-Industry-Corporation of Regional Development
Palabras Clave: Universidad - empresa - región, desarrollo humano, cooperación.

Keywords: University-company-region, human development, cooperation.

\section{Resumen}

Esta ponencia presenta en primer lugar, una experiencia de trabajo entre universidades, industrias y una corporación de desarrollo, integradas desde el año 2007 en el proyecto Red Bolivariana de Educación Superior-CVG. Esta red desarrolla programas de formación dirigidos a trabajadores de la Corporación Venezolana de Guayana e industrias básicas, que son pertinentes con las orientaciones estratégicas de la corporación y de las industrias.

Posteriormente, se presenta la propuesta de creación de un Centro de Estudios Territoriales. Este centro representa la 2da etapa de la Red, el cual permitirá la investigación y formación a niveles de postgrado para el desarrollo de líneas que atiendan el estudio de la problemática regional, y el análisis y evaluación de las estrategias y políticas dirigidas a la región Guayana con el objetivo de incidir en las transformaciones territoriales que hagan posible un Desarrollo Humano, Endógeno, Desconcentrado y Sustentable de la Región (CVG-Red de Educación Superior, 2007:1).

La propuesta del Centro se inspira, desde el punto de vista académico-social, en los esfuerzos y aportes de diferentes redes sociales de investigadores cuyo objeto de estudio son los procesos que atañen al territorio, entre las que se
1 Ponencia que se presenta al VIII Encuentro de Programas de Postgrados Iberoamericanos sobre desarrollo regional y políticas territoriales (RIPPET). Manizales Colombia, 6 al 8 de Agosto de 2009.

2 Socióloga UCAB. MSc. Planificación del Desarrollo. Mención Regional-Urbana. CENDES-UCV. Doctora en Estudios del Desarrollo. CENDES-UCV. Coord. Red de Educación Superior-CVG. Región Guayana-Venezuela. $\mathrm{E} \quad \mathrm{m} \quad \mathrm{a}$ i $\quad l$ : correo.nancyac@gmail.com. 
cuentan: la Red Iberoamericana de Investigadores sobre Globalización y Territorio (RII), IV Simposio Territorios y Sociedades en un Mundo en Cambio, Red Nacional de Investigación Urbana (RNIU) de México y la Red Iberoamericana de Postgrados sobre Políticas Territoriales Estudios Territoriales (RIPPET).

\begin{abstract}
This paper aims primarily at showing an experience of collaborative effort among universities, industries and municipal development corporations. Since 2007, these three institutions integrated the project "Red Bolivariana de Educación Superior - CVG". This network develops training programs for employees of the Venezuelan Corporation of Guayana (CVG) and basic industries, which are relevant and meet the strategic guidelines of the development corporations and industries.
\end{abstract}

Secondly, this paper presents a proposal for the creation of "The Center for Territorial Studies". This center represents the second phase of the abovementioned Network, which will offer research and training at a graduate level for the development of research lines. This research involves regional problems as well as the analysis and evaluation of strategies and policies oriented to the Guayana region. This has the objective of influencing the transformations of the territory to favor a human, endogenous, decentralized and sustainable development of the region (CVG-Red de Educación Superior, 2007:1).

The proposal of the Center is, from an academic-social perspective, based on the efforts and contributions of different social networks of researchers. Their object of study is the process pertaining to the territory, namely, Iberoamerican Network of Researchers on Globalization and Territory (RII), IV Symposium on Territories and Societies in a Changing World, National Network of Urban Investigation (RNIU) of Mexico and the Iberoamerican Network of Graduate Studies on Policies and Territorial Studies (RIPPET).

\section{Antecedentes:}

El proyecto Red de Educación Superior se inicia a partir del convenio entre Ministerio Popular para la Educación Superior (MPPES) y la Corporación Venezolana de Guayana (CVG). ${ }^{3}$ Corporación que tiene la responsabilidad del desarrollo de la Región Guayana y otros Estados del país que representan en cuanto al territorio venezolano, el $62 \%$ del mismo. Espacio geográfico fuente de materia prima tales como bauxita, hierro, agua, bosques y oro que lo hace la región más rica del país. Se desarrolla como complejo industrial a partir de la propuesta de Polos de Desarrollo de Perroux creándose un centro urbano industrial: Ciudad Guayana y un programa regional.

Dicho proyecto surge para fortalecer la gestión de la corporación de desarrollo regional que además es casa matriz de un conjunto de empresas básicas de los sectores del aluminio, hierro-acero, hidroelectricidad, minería, forestal y oro.
3 Se suscribe el convenio entre estas dos instituciones en el año 2006 dando inicio a los programas de formación en el mes de enero del año 2007.

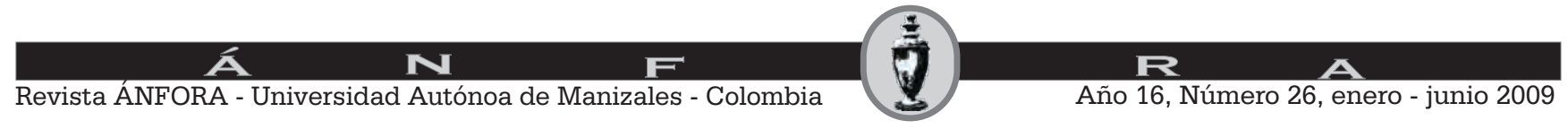


Está orientado a establecer las alianzas estratégicas entre la CVG, las Instituciones de Educación Superior, Empresas del Estado y otros actores para la formación, investigación y gestión territorial.

En este sentido, se ha dedicado en sus primeros años a atender la formación de los trabajadores para elevar sus niveles de conocimientos en función de la gestión y desarrollo del territorio. A medida que se han desarrollado los programas de formación han surgido las demandas en otras poblaciones, indígenas y semi-rural que han sido incorporadas también, partiendo de la convicción que si bien los programas de formación están dirigidos a los trabajadores no podemos excluir otros grupos sociales que no han tenido acceso a la educación superior. Debemos contribuir con el logro de la equidad para hacer posible el desarrollo humano, endógeno, desconcentrado y sustentable de la región (CVG-Red de Educ Superior, 2007:1).

\section{LINEAMIENTOS QUE ORIENTAN LA ACCIÓN DE LA RED DE EDUCACIÓN SUPERIOR.}

La gestión del proyecto y los programas de formación se inscriben en las Líneas Generales del Plan Nacional Simón Bolívar 2007-2013 (MPPPD, 2006): Nueva Ética Socialista, Suprema Felicidad Social, Democracia Protagónica Revolucionaria, Modelo Productivo Socialista y Nueva Geopolítica Internacional. Entre sus principales orientaciones están:

\section{Nueva Ética Socialista.}

Se plantea con este mandato trabajar por la refundación ética y moral basada en los valores humanistas del socialismo para la superación del espíritu capitalista.

El compromiso del proyecto de la Red es promover la inclusión de los principios éticos de solidaridad humana elevando el nivel de formación y conciencia de los trabajadores y comunidades para su participación en los procesos de transformación a nivel regional, local que deben estar fundamentado en principios y valores.

\section{Suprema Felicidad Social.}

Un segundo reto es la construcción de una estructura social incluyente formando un nuevo modelo social, productivo, socialista, humanista y endógeno.

La Red con el fin de contribuir con las transformaciones hacia una región socialmente incluyente, desarrolla las capacidades humanas de los trabajadores de la región, lleva a cabo proyectos socio-ambientales en las comunidades y sectores productivos y atiende comunidades indígenas para el fortalecimiento de su identidad cultural.

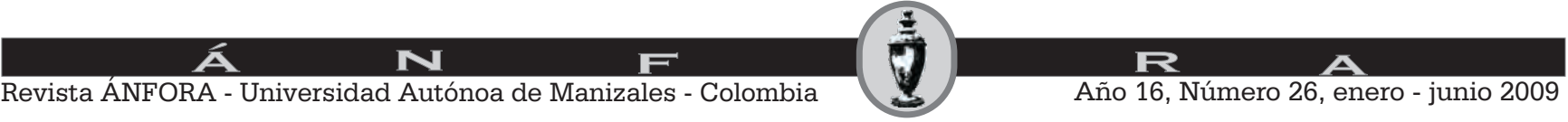




\section{Democracia Protagónica Revolucionaria.}

Con el fin de fortalecer los principios de la organización y participación fundamentados en la cooperación y solidaridad, la Red trabaja en las comunidades a través de los proyectos de los estudiantes, e incrementa los niveles de conocimiento del funcionario público (Nuevo perfil del servidor público) y facilita el acceso de los trabajadores a la utilización de los medios de comunicación social de la región para desarrollar temas de formación, participación ciudadana y divulgación de derechos, deberes e identidad nacional.

\section{Modelo Productivo Socialista.}

Participa en los esfuerzos por transformar el modelo productivo fomentando el desarrollo de trabajos en los estudiantes hacia los estudios diagnósticos, solución de problemas y propuestas socio-productivas. Así como en la creación de una red de intercambio de información entre Centros de Educación y Empresas para el desarrollo de investigaciones y programas de formación requeridos para la construcción de un nuevo modelo socio-productivo.

\section{Nueva Geopolítica Internacional.}

La Red se inscribe en los lineamientos de integración latinoamericana y caribeña y la diversificación de las relaciones internacionales participando bajo relaciones de cooperación con Instituciones de América Latina y el Caribe para el desarrollo de programas de formación e investigación que contribuyan a la identidad y autodeterminación de los pueblos.

En la actualidad atiende la formación en estudios universitarios en: ambiente, social, agro-ecología, educación y en las áreas tecnológicas como sistemas industriales, mecánica y electricidad. Ello con el objetivo de contribuir en la formación universitaria con programas pertinentes para la gestión del territorio y dentro de éste en la conformación de un nuevo modelo socio-productivo.

Las dos poblaciones a las cuales preferentemente se orientan estos programas son los trabajadores de CVG y empresas; y las comunidades indígenas, con el objetivo de dar respuesta a aquellas poblaciones con mayores dificultades para incorporarse a los programas de formación, bien por las exigencias del trabajo productivo o por las condiciones geográficas que dificultan el acceso hacia las comunidades indígenas.

Participan trabajadores de la mayor parte de las empresas básicas: CVGBAUXILUM, CVG-FERROMINERA DEL ORINOCO, EDELCA, CVG-PROFORCA, CVG-VENALUM y de la Corporación de Desarrollo -CVG.

En el caso de las empresas, éstas tienen como objetivos la explotación de hierro, generación de hidroelectricidad, explotación y tratamiento de la bauxita para su transformación en aluminio, cultivo y aprovechamiento de pinos. Se tienen como ámbitos de acción: Ciudad Guayana, ciudad sede del complejo industrial y dos comunidades: semirural e indígena, ubicadas en áreas geográficas en las

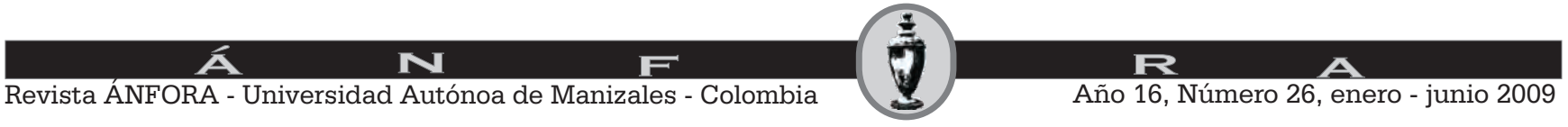


cuales se desarrollan proyectos de generación hidroeléctrica y de conservación ambiental por parte de la CVG y EDELCA.

Las instituciones universitarias que participan en el proyecto El Instituto Universitario Tecnológico del Estado Bolívar (IUTEB) y la Universidad Bolivariana de Venezuela. (UBV), ${ }^{4}$ y la Universidad Nacional Experimental de Guayana (UNEG). ${ }^{5}$ Ésta última en la definición de líneas conjuntas de investigación.

Una vez iniciado los programas de formación se evidencia la necesidad de avanzar con las funciones de investigación y postgrado dadas las exigencias de conocimientos y de estudios sistemáticos para el abordaje de la problemática regional, de las políticas que se implementan en la actualidad y aquellas que se requerirían para lograr el desarrollo humano, endógeno, desconcentrado y sustentable de la Región.

\section{PROPUESTA PARA LA CREACIÓN DE UN CENTRO DE ESTUDIOS TERRITORIALES.}

En la actualidad se trabaja bajo el enfoque de participación de actores: universidad, empresas y corporación, en las fases de búsqueda de viabilidad política, institucional y técnica. En este trabajo se presentan sólo los aspectos referidos a la viabilidad técnica: objeto de estudio, la red de problemas a los cuales se orientaría la indagación sobre los procesos territoriales y las principales líneas de investigación y formación.

\section{Objeto de estudio y red de problemas:}

Con esta propuesta se aspira responder a las necesidades de indagación del contexto regional y local con el objetivo de contribuir en la gestión de la corporación de desarrollo, de las empresas básicas y otras instituciones con responsabilidades en el desarrollo de la región y sus centros poblados y que tienen como directriz impulsar cambios en el modelo de desarrollo industrial, urbano y regional que se inscribió en la propuesta de Polos de Desarrollo en los años 60 y que posteriormente durante los años 80 fue objeto de la aplicación de políticas neoliberales orientadas hacia la reestructuración económica y políticas.

El programa de industrialización básica en Venezuela a inicio de los años 60 fue diseñado por el Estado como un modelo de desarrollo regional fundamentado en la industrialización por sustitución de importaciones impulsada por los gobiernos nacionales para América Latina desde los años 40-50. Esta experiencia para el caso de la región Guayana ha sido reconocida como un enclave industrial por su poco dinamismo hacia el ámbito regional y por la inexistencia de encadenamientos productivos que agregaran valor a la materia prima y favorecieran la transformación productiva.

Sin embargo condujo a una dinámica específicamente en Ciudad Guayana de altas inversiones, importantes procesos migratorios (Acosta, 1986) y una
4 El IUTEB y la UBV son instituciones universitarias creadas en los últimos 10 años que comparten las orientaciones de desarrollo para el país así como la filosofía educativa de vinculación con el entorno para su transformación.

5 La UNEG es la Universidad Regional creada en la década de los 80 y con una filosofía de gestión orientada a la búsqueda de la pertinencia regional.

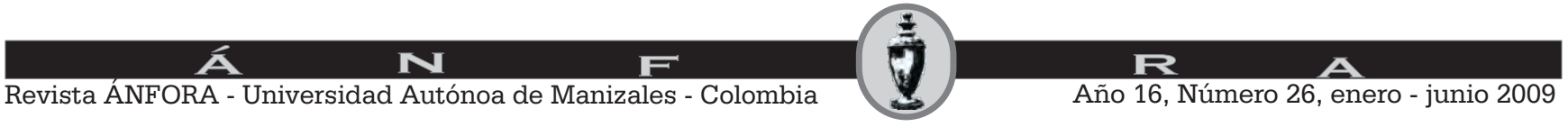


dinámica sociopolítica impulsada por la creación de un importante movimiento sindical a la par de un predominio del Estado en la gestión económica-socialambiental y política.

Posteriormente con las políticas de apertura económica y política llevadas a cabo en América Latina y específicamente en Venezuela se lleva adelante el programa de privatización con una intensa dinámica sociopolítica, así como también un proceso de descentralización que favoreció específicamente en Ciudad Guayana (sede del programa industrial) la emergencia de actores locales cuyo origen estuvo en los grupos sindicales surgidos con la dinámica industrial (Acosta, 2004).

Ambos momentos, presentados brevemente en los párrafos anteriores: 1. Un modelo de industrialización básica con predominio del Estado y 2. Reestructuración económica y política que condujo al debilitamiento del Estado, han desencadenado procesos de carácter económico y sociopolíticos relevantes con impactos en el modelo regional-local.

En los últimos diez años se ha planteado para Venezuela y la región a través de los Planes para el Desarrollo Económico y Social 2001-2007 y 2007-2013 y el Plan Nacional de Desarrollo Regional 2001-2007; y de un conjunto de políticas, programas cambios estratégicos en el modelo de organización, desarrollo y gestión del país.

En el Plan 2001-2007 se plantea orientar los esfuerzos del país hacia el logro de cinco equilibrios: económico, social, político, territorial e internacional (Ministerio de Planificación, 2001). En el equilibrio territorial se plantea como objetivo determinante ocupar y consolidar el territorio por la vía de descentralizar pero también desconcentrar población, inversiones y recursos que históricamente se han consolidado en el eje Norte Costero por el modelo de desarrollo económico que condujo a la concentración en este eje, lo que hace indispensable modificar el patrón de poblamiento a través de la diversificación y consolidación de la economía en todos los espacios con potencial de desarrollo

Para avanzar en el logro de este objetivo se plantea como necesario la diversificación y consolidación de la economía en todos los espacios con potencial de desarrollo. Se definen territorialmente tres Ejes de Desconcentración: Orinoco-Apure, Oriental y Occidental ubicando estratégicamente como objeto del desarrollo ámbitos del territorio con potencialidad productiva pero históricamente despoblados y sin aprovechamiento económico y que deben dar como resultado una nueva ordenación del territorio superando así los desequilibrios territoriales.

Estos Ejes a su vez son fundamentales en las estrategias para el equilibrio internacional por cuanto se vinculan a las consideradas fachadas de integración contenidas en los lineamientos de desarrollo: Amazónica, Andina y Caribeña con las cuales se hace el diseño y ejecución de planes de desarrollo en las ciudades fronterizas y las políticas de integración, concertación y cooperación latinoamericanas (Ministerio de Planificación y Desarrollo, 2001: 9). 
Las nuevas estrategias de desarrollo territorial a nivel regional-local se orientan al desarrollo desconcentrado y equilibrado en el territorio para lo cual se plantea como objetivos estimular armónicamente los aspectos económicos, sociales, políticos y culturales de colectivos, fundamentalmente de aquellos que han sido excluidos. Para ello motiva la participación de la comunidad en la planificación de la actividad económica apoyándose en las capacidades para dar respuesta a las necesidades y garantizar calidad de vida. Promueve los valores de cooperación y solidaridad, así como el trabajo productivo y el equilibrio ecológico.

La estrategia para el desarrollo del nuevo modelo territorial está basada en la generación de Núcleos de Desarrollo Endógeno (NUDES). Los cuales se corresponden con un ámbito territorial definido y seleccionado para la promoción y desarrollo del Nuevo Modelo Socio - Productivo, a partir del aprovechamiento sustentable de sus potencialidades naturales y humanas, bajo esquemas de organización social asociativos y participativos, para la producción de bienes y servicios, que permitan mejorar la calidad de vida de sus habitantes.

La estrategia socio-productiva está basada en la conformación de Empresas de Producción Social (EPS) como células fundamentales de nuevas relaciones de producción y propiedad y orientada a la producción de bienes y servicios que satisfagan necesidades básicas de la comunidad (CVG, 2006: Dir. Res. 9137)

Los Núcleos de Desarrollo Endógeno (NUDES) y las Empresas de Producción Social (EPS) son unidades fundamentales del modelo endógeno basado en relaciones socio-productivas con las cuales se aspira generar condiciones de vida dignas para la población y que en el contexto del avance de los ejes de desconcentración hagan posible un desarrollo territorial que transforme aquellos espacios del país en la cual existe población que ha estado excluida de la vida económica, social y política.

Asimismo se ha planteado que a las industrias básicas conviertan a partir de sus procesos más importantes e incorporación de EPS en Núcleos Endógenos Industrializantes y en la actualidad se avanza hacia la búsqueda de un modelo productivo socialista.

El modelo de desarrollo endógeno ha estado acompañado por una estrategia de inclusión social y productiva, la cual consiste en un conjunto de políticas y acciones concebidas e integradas en red, orientadas a cancelar la deuda social que el Estado representativo mantiene con la sociedad. Por esta vía se ha atendido en las áreas de salud, educación, identidad, capacitación, participación y organización socioproductiva a los sectores sociales históricamente excluidos y en situación de pobreza. En especial, las misiones sociales se han constituido en un instrumento para superar el Estado burocrático, ineficiente y excluyente y transformarlo en un Estado de justicia, eficiente, eficaz e inclusivo. 
Para el país se han propuesto un conjunto de proyectos nacionales que tienen como objetivo integrarlo territorialmente, lo cual implica para el nivel nacional, regional y local el aumento de las posibilidades de comunicación y por ende de integración entre poblados y creación de las condiciones para los cambios en la economía regional y local. Estos proyectos son los planes nacionales: Ferroviario, de Telecomunicaciones, Gas, Transporte Fluvial y Corredor Vial Transguayana con un importante impacto en la región Guayana la cual tiene aprox. El 52\% del territorio venezolano y áreas desatendidas de medios de comunicación.

Dada la condición de la Corporación como ente de desarrollo, los cambios producidos en el entorno regional e institucional, y en las orientaciones para profundizar en los cambios que requiere el país y la región se considera necesario y pertinente la creación de un Centro en Estudios Territoriales que haga posible la mirada crítica de lo que ha sido el modelo de desarrollo hacia la región en el pasado ${ }^{6}$ pero asimismo, una mirada crítica hacia la implementación e impactos de las políticas y programas actuales de manera que contribuya con la gestión del Estado Venezolano y la Corporación proporcionándoles análisis, evaluación y propuestas que faciliten el avance hacia la situación deseada en materia de desarrollo territorial.

En este mismo sentido, se propone con la existencia del Centro realizar esfuerzos sistemáticos para lograr un conocimiento pertinente que describa y explique la realidad de la región y que esté orientado por valores de solidaridad, compromiso, equidad, justicia social, libertad, responsabilidad y disciplina, es decir una ética del conocimiento, o como plantea Boisier (2008: 124-128): una epistemología y axiología sobre gestión del desarrollo territorial.

\section{Las líneas de investigación/ejes de formación.}

La propuesta del Centro de Estudios Territoriales se fundamenta desde el punto de vista teórico en una concepción del territorio como la expresión de relaciones sociales y procesos socio-económicos, socio-políticos y socioculturales plasmados en un ámbito local, regional o nacional en articulación con procesos internacionales y globales, en cuyas transformaciones cumple un importante papel los actores sociales. En este sentido se pueden visualizar las transformaciones territoriales (Acosta,2004: 50): como "construcción social" (Alburquerque, 1997),...una lógica diferencial desde el punto de vista económico basado en la emergencia de nuevas creaciones económicas (Madoery, 2001:25) y una territorialidad como capacidades productivas, sociales, políticas y culturales en contraposición a la fragmentación y desterritorialización producida por el capital (Castells, 1986:23 citado por de Mattos, 1990:236).

Desde el punto de vista académico-social se consideran las contribuciones que realizan diferentes redes científicas de investigadores cuyo objeto de estudio es el territorio: Red Iberoamericana de Investigadores sobre Globalización y Territorio (RII), IV Simposio Territorios y Sociedades en un Mundo en Cambio,
$6 \mathrm{Al}$ respecto es de interés el análisis de las políticas y sus efectos territoriales que es analizado por González L. (2008) en Políticas e Instituciones para el Desarrollo Económico

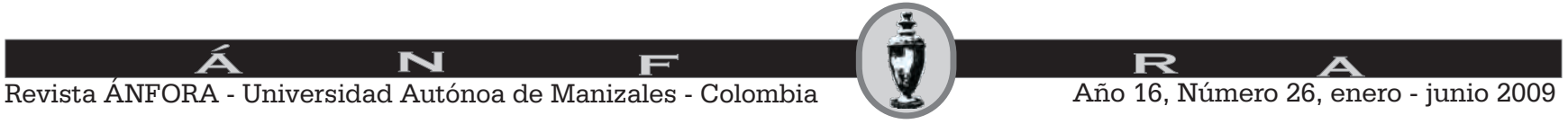


Encuentro de la Red Nacional de Investigación Urbana (RNIU) de México y la Red Iberoamericana de Postgrados sobre Políticas Territoriales Estudios Territoriales (RIPPET).

Las Líneas de Investigación y Ejes de Formación constituyen una misma matriz que se retroalimentan mutuamente. Se formulan considerando los avances en investigación y programas de formación a nivel latinoamericano, así como las políticas y programas y sus efectos actuales e impactos territoriales futuros evaluando su contribución y limitaciones en términos de la visión prospectiva que se tiene para la región y su transformación territorial, considerando las líneas de desarrollo nacional y que son a su vez orientaciones para el desarrollo a nivel territorial: regional-estadal-local en las dimensiones sociales, económicas, ambientales y políticas entendiendo la organización del territorio como expresión de los procesos económicos, sociales y políticos en el cual los actores tienen un papel protagónico.

Los ejes de formación se concretan en tres componentes o contenidos principales: teórico-metodológicos, instrumentales y ético-políticos (Ver González López, 2005: 19): los conceptos, teorías y metodologías que permiten describir y, sobre todo, explicar, los procesos territoriales y sus resultados, los elementos que permiten aplicar los conocimientos a la realidad para que, con base en las explicaciones de los procesos, sea posible producir modificaciones en razón a las explicaciones y orientaciones provenientes de los componentes ético-políticos.

Cada uno de estos ejes supone el estudio y comprensión de distintas disciplinas que se integran en una malla curricular para alcanzar los objetivos en cada uno de estos ejes:

\section{Axiológico:}

La importancia de los valores para la transformación del territorio.

\section{Epistemológico:}

Necesidad de un paradigma para la comprensión de lo territorial. Estudio de las diferentes teorías sobre la organización y transformación del territorio. Orientaciones teóricas-metodológicas en la interpretación del territorio. Construcción de un paradigma para la comprensión de lo territorial. Interdisciplinariedad y Transdisciplinariedad.

\section{Socio-productivo:}

Diagnóstico y comprensión de experiencias industriales en términos de modelos correspondientes con períodos de crecimiento industrial y políticas nacionales. Estudio del Arte de experiencias e impactos territoriales. Análisis de experiencias y propuestas de modelos socioproductivos alternos en el cual se consideren: las transformaciones de las relaciones sociales con el objeto y proceso de producción. El manejo de tecnologías productivas y limpias y el uso

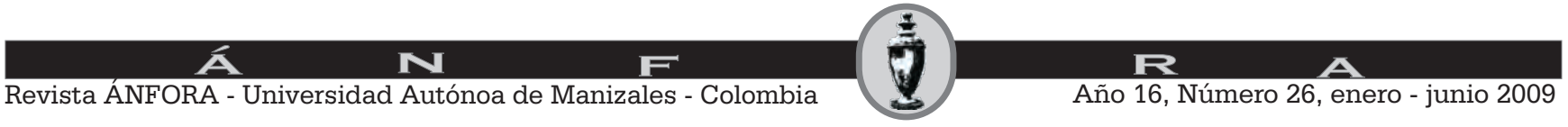


de recursos bajo condiciones sustentables. Articulación a otras formas sociales de organización económica.

\section{Socio-comunitario:}

Comprensión de la dinámica poblacional en la ocupación del territorio. Factores que inciden los procesos de desconcentración económica para el aprovechamiento de potencialidades de manera socialmente sustentable. Análisis y propuestas de Desarrollo Endógeno a nivel regional y local. Situación social y económica de las ciudades y principales centros poblados. Nuevas orientaciones para el desarrollo de las ciudades. Poblaciones indígenas y territorio. Proyectos e impactos territoriales en términos Socioculturales y Ambientales.

\section{Socio-político:}

Estudios de las teorizaciones políticas que contribuyan en la identificación y comprensión de los actores sociopolíticos: internacionales, nacionales, territoriales y sus dinámicas. La Gestión Pública. Papel del Estado en la configuración del territorio. Viejos y Nuevos actores territoriales. Formas de organización y participación.

\section{Socio-Cultural:}

Diagnósticos sobre las condiciones de las poblaciones indígenas en la región. Propuesta para el fortalecimiento de la Interculturalidad y arraigo de las poblaciones indígenas. Levantamiento del patrimonio cultural regional. Cultura urbana.

\section{Socio-Ambiental:}

Situación e impacto ambiental producido por los procesos de producción. Estudio y elaboración de propuestas sobre economía ecológica. Situación actual en el manejo de los recursos naturales y propuesta para el manejo sustentable.

\section{Instrumental:}

Inventario, análisis y comprensión de los instrumentos para conocer e incidir sobre el territorio. Implicaciones teóricas-metodológicas y prácticas de su aplicación. Metodologías cualitativas y cuantitativas. Planificación: ordenamiento territorial, planes de desarrollo, proyectos, planificación participativa; Cartografía social, Sistemas de Información Geográfica; Evaluación de Impactos; Metodologías de intervención social; estudios de laboratorios, simulaciones. Observatorios industriales, económicos y sociales. Mapas, croquis e imágenes. 

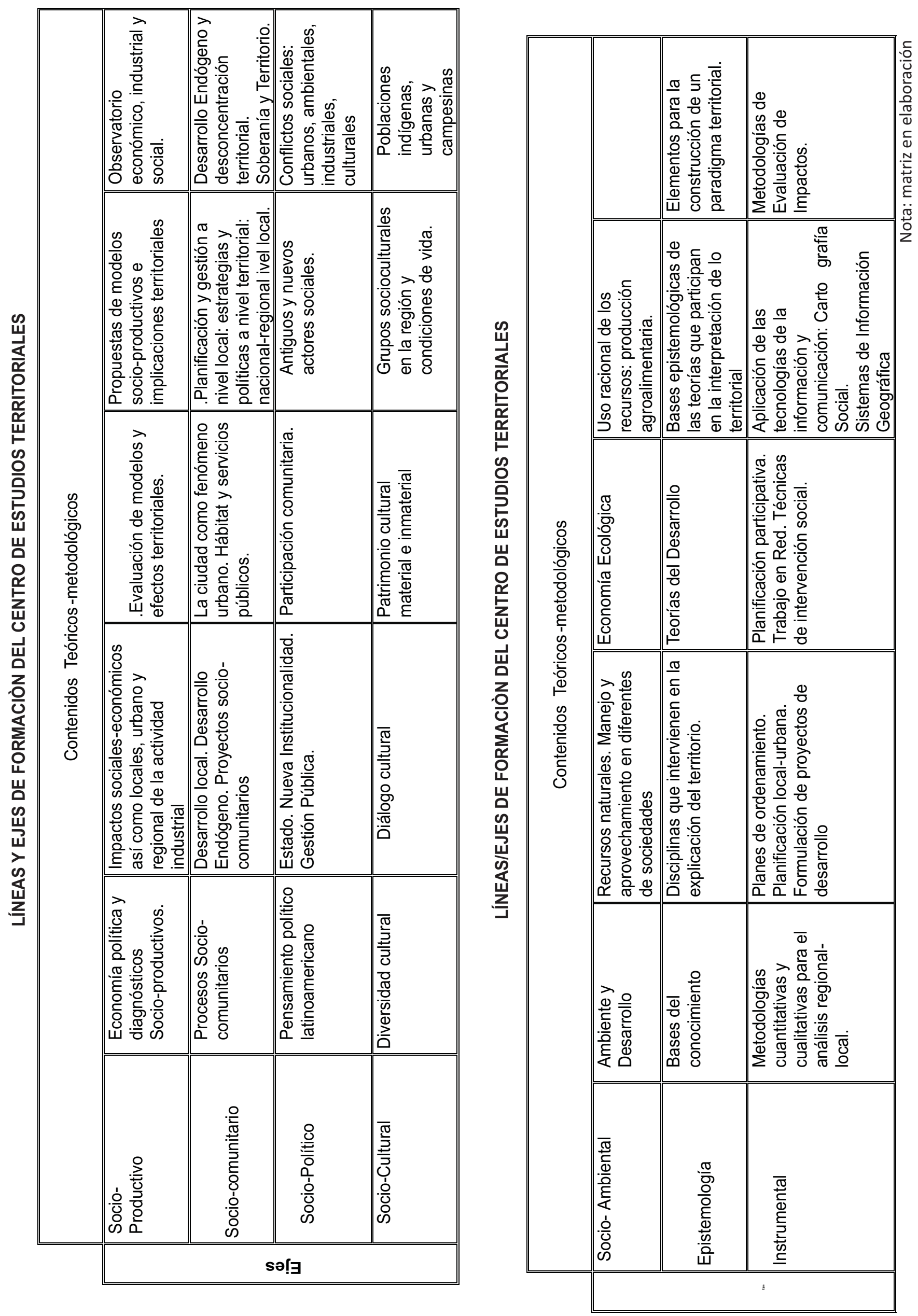
Para el desarrollo de esta propuesta es de vital importancia:

- Conformar equipos de estudios orientados a atender las diferentes áreas de investigación y formación.

- Contribuir en la conformación de una base de datos a nivel iberoamericano sobre los programas de formación e investigación en territorio: en lo social, económico, político, ambiental y geográfico, así como en las metodologías de investigación en el tema.

- Proponer y participar en líneas y proyectos integrados por investigadores de distintos centros, universidades y países.

- Crear base de datos o blogs sobre el tema territorial: en los aspectos epistemológicos y paradigmáticos: integración de disciplinas orientados a las transdisciplinariedad.

- Participación en foros e intercambios entre países y centros de investigación. Postdoctorados.

- Cursos en conjunto en los temas: teórico-metodológico en desarrollo, planes urbanos, planes de ordenamiento territorial, prospectiva, participación comunitaria, sistemas de información geográfica. Ellos pudieran combinar estrategias presenciales con virtuales.

- Establecer como etapa inicial una oferta universitaria de universidades latinoamericanas que respondan a las demandas de estudios en la Región y que esté fundamentada en relaciones de cooperación. Trabajar con estrategias semi presenciales y virtuales y con la conformación de un equipo regional que asegure la contextualización de los estudios

\section{Bibliografía}

ACOSTA, N. (1986). La política social de la Corporación Venezolana de Guayana (CVG) y los sectores populares de San Félix. Trabajo de grado de maestría. Centro de Estudios del Desarrollo. Universidad Central de Venezuela, Caracas.

ACOSTA, N. (1997, enero-abril). Los cambios en la realidad regional: Implicaciones para la planificación y el desarrollo regional. Parima, revista de ciencias sociales de Guayana, 1, 4-32, Ciudad Guayana.

Acosta N. y Jungemann, B. (2000, enero-abril). Transformación territorial

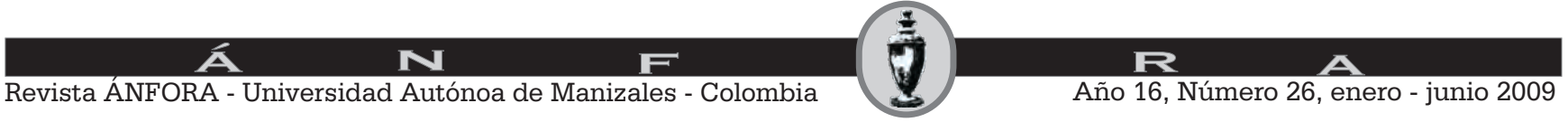


y gestión local: el caso de Ciudad Guayana. En Globalización, reestructuración y transformación territorial, 43, 17, 121-154. Cuadernos del CENDES. Caracas.

ACOSTA, N. (2004).Reestructuración económica y política y su impacto territorial: el caso de Ciudad Guayana. Trabajado de grado de Doctorado. Centro de Estudios del Desarrollo. Universidad Central de Venezuela, Caracas.

ACOSTA, N. (2006). Nuevas dinámicas económicas y sociopolíticas, cambios en el Estado y transformaciones en el modelo regional-local: el caso de la región Guayana. Venezuela. Ponencia presentada en el IX Seminario Internacional de la Red Iberoamericana de Investigadores sobre Globalización y Territorio. Bahía Blanca. Argentina.

Alburquerque, F. (1997). El proceso de construcción social del territorio para el desarrollo económico local. Instituto Latinoamericano del Caribe de Planificación Económica y Social. Chile.

BOISIER, S.(2007) Imágenes en el Espejo. Aportes a la discusión sobre Crecimiento y Desarrollo Territorial. Universidad Autónoma del Estado de México-UAEM. Toluca, México. .

BORJAS, J. (2001). El gobierno del territorio de las ciudades latinoamericanas. Disponible: me//C: \Artículo Borjas.htm [consulta julio 2, 2001]

CALDERÓN, F. y Santos, M. dos (1988). Hacia un nuevo orden estatal en América Latina. Buenos Aires: Centro Latinoamericano de Ciencias Sociales.

CORAGGIO, J. L. (2001). La promoción del desarrollo económico en las ciudades: el rol de los gobiernos municipales. Ponencia presentada en la Reunión Anual de Trabajo Red N 5 "Políticas Sociales Urbanas", del Programa URB-AL. Montevideo.

Corporación Venezolana de Guayana (2006). Resoluciones de Directorio No 3791 de la Corporación Venezolana de Guayana.

GONZÁLEZ LÓPEZ, S. (2005). Posgrados en Estudios Territoriales: entre la competencia y la cooperación. Ponencia presentada en el VI Encuentro de Postgrados Iberoamericanos sobre Desarrollo y Políticas Territoriales: "construyendo espacios para la colaboración regional. Toluca, México, 19 al 21 de septiembre de 2005.

GONZÁLEZ LÓPEZ, S. (2008). Políticas e Instituciones para el Desarrollo Económico Territorial (DET). ILPES. Santiago de Chile. 2008

JUNGEMANN, B. (1996). Desarrollo regional y descentralización en América Latina en el marco del ajuste: una relación con muchas interrogantes. 32, año 13, 61-95. Cuadernos del CENDES, Caracas.

JUNGEMANN, B. (2002, mayo-agosto). Transformaciones territoriales y socioterritoriales en la globalización. 50, año 19, 155-163. Cuadernos del CENDES. Caracas.

MADOERY, O. (2001). El valor de la política de desarrollo local. En Vázquez Barquero y O. Madoery. En transformaciones globales,

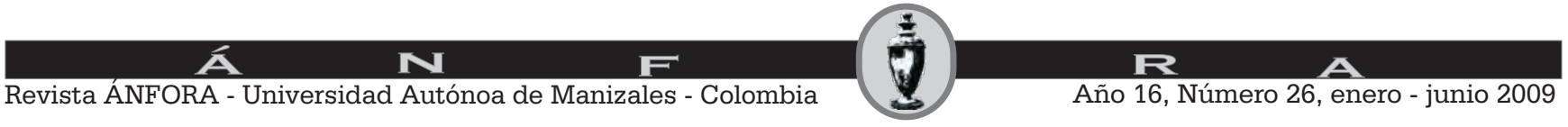


instituciones y políticas de desarrollo local. Edic. Homo Sapiens. Rosario.

MATTOS, C. de (1990). Reestructuración social, grupos económicos y desterritorialización del capital: El caso del Cono Sur. En F. Alburquerque, C. Mattos de y R. Jordan (Comps.) Revolución tecnológica y reestructuración productiva: Impactos y desafíos territoriales. Buenos Aires: Instituto Latinoamericano del Caribe de Planificación Económica y Social /Organización de las Naciones Unidas/ Instituto de Estudios Urbano de la Pontificia Universidad Católica de Chile.

MINISTERIO DE PLANIFICACIÓN Y DESARROLLO (2000). Líneas Generales del Plan de Desarrollo Económico y Social de la Nación 20012007.

MINISTERIO DE PLANIFICACIÓN Y DESARROLLO (2001). Plan Nacional de Desarrollo Regional 2001-2007.

MINISTERIO DE PLANIFICACIÓN Y DESARROLLO (2006). Líneas Generales del Plan de Desarrollo Económico y Social de la Nación 20072013.

PRESIDENCIA DE LA REPÚBLICA BOLIVARIANA DE VENEZUELA (2005): La nueva etapa. El nuevo mapa estratégico. Edic. Imprenta universita de la Universidad Bolivariana de Venezuela. Caracas.

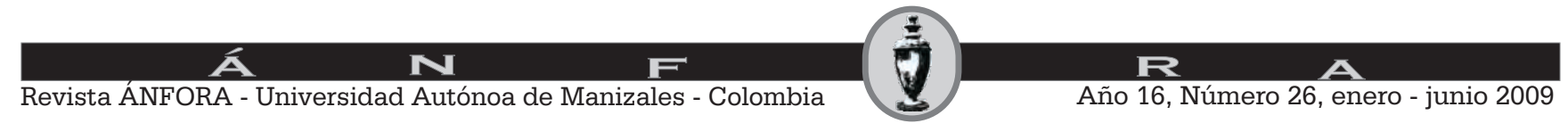

